\title{
Products of shifted primes: Multiplicative analogues of Goldbach's problem
}

\author{
by
}

P. D. T. A. Elliott (Boulder, Colo.)

\section{I begin with}

CONJECTURE I. If $N$ is a sufficiently large positive integer, then every rational $r / s$ with $1 \leq r \leq s \leq \log N,(r s, N)=1$, has a representation of the form

$$
\frac{r}{s}=\frac{N-p}{N-q}, \quad p, q \text { prime }, p<N, q<N .
$$

The case $r=1$ is equivalent to solving $(s-1) N=s x-y$ in positive primes $x, y$ not exceeding $N$. Goldbach's problem is to correspondingly solve $N=x+y$.

CONJECTURE II. There is a positive integer $k$ so that in the above notation and terms there are representations

$$
\frac{r}{s}=\prod_{i=1}^{k}\left(N-p_{i}\right)^{\varepsilon_{i}}, \quad \varepsilon_{i}=+1 \text { or }-1,
$$

with the primes $p_{i}$ not necessarily distinct.

CONJECTURE III. There are representations of this type, but with the number, $k$, of factors needed possibly varying with $r$ and $s$.

An ideal method? Consider first the problem of representing 2 in the form $(p+1)(q+1)^{-1}$ with primes $p, q$, an analogue of the prime-pair problem.

Let $Q^{*}$ denote the multiplicative group of positive rationals. The dual group $\widehat{Q^{*}}$ may be identified with the (direct) product of denumerably many copies of $\mathbb{R} / \mathbb{Z}$. It is rather "large". A typical character $g: Q^{*} \rightarrow U$ (-nit circle in $\mathbb{C}$ ) is, in classical parlance, a unimodular complex-valued completely multiplicative arithmetic function. There is a translation invariant Haar measure $d \mu(g)$ on $\widehat{Q^{*}}$ that assigns to the whole (compact) group measure 1.

1991 Mathematics Subject Classification: Primary 11N05; Secondary 11N99, 11L99.

Partially supported by NSF contract DMS 9530690. 
We choose a weight $w_{p}$ so that $S(g)=\sum w_{p} g(p+1)$, taken over all primes $p$, converges absolutely, uniformly for $g$ in $\widehat{Q^{*}}$. Then there is a representation

$$
\sum_{2=(p+1) /(q+1)} w_{p} \bar{w}_{q}=\int_{\bar{Q}^{*}} g(2)|S(g)|^{2} d \mu(g) .
$$

To study the Goldbach analogue in Conjecture I we replace $Q^{*}$ by $Q_{1}$, the group generated by the primes $p$ not exceeding $N,(p, N)=1$, and $S(g)$ by $\sum g(N-p)$ taken over the same primes. We may naturally restrict $d \mu(g)$ to $\widehat{Q}_{1}$, and $\int_{\widehat{Q}_{1}} g(2)|S(g)|^{2} d \mu(g)$ represents the number of solutions to $2=(N-p)(N-q)^{-1}, p, q \leq N,(p q, N)=1$. In standard notation, $\widehat{Q}_{1}$ is $(\mathbb{R} / \mathbb{Z})^{\pi(N)-\omega(N)}$; convergence properties are not needed, but an explicit dependence of the integral upon the parameter $N$ is introduced.

Consider the analogous representation in the Hardy-Littlewood circle method. There the rôle of $Q_{1}$ is played by $\mathbb{Z} . \widehat{\mathbb{Z}}$ may be identified with $\mathbb{R} / \mathbb{Z}$, and a typical character $g_{\alpha}$ on $\mathbb{Z}$ is given by $n \mapsto \exp (2 \pi i \alpha n)$, where $\alpha(\bmod 1)$ is fixed. If $Y(\alpha)=\sum \exp (2 \pi i \alpha p)$, taken over the primes $p \leq N$, then $\int_{\widehat{\mathbb{Z}}} \exp (-2 \pi i \alpha N) Y(\alpha)^{2} d \alpha$ is the number of solutions to $N=p+q$, with $p, q$ prime.

We cannot currently estimate this integral satisfactorily, but its analogue with $Y(\alpha)^{3}$ in place of $Y(\alpha)^{2}$ we can. Following the standard procedure the interval $[0,1)$ (i.e., the group $\widehat{\mathbb{Z}}$ ) is decomposed into major and minor arcs. The major arcs are (small) intervals around rationals $a k^{-1}(\bmod 1)$, with $(a, k)=1, k$ "small compared to $N$ ". To view this group-theoretically, define a (translation invariant) metric $\sigma$ on $\widehat{\mathbb{Z}}$ by $\sigma\left(g_{\alpha}, g_{\beta}\right)=\|\alpha-\beta\|=$ $\min |\alpha-\beta-m|$, the minimum taken over all integers $m$. The major arcs are then the union of spheres $\left(g ; \sigma\left(g, g_{t}\right) \leq \delta\right)$ around characters $g_{t}$ with $t$ rational, of small denominator $k$. In particular $g_{r}^{k}=1$, i.e. the characters $g_{r}$ are of order low compared to $N$.

What remains of $\widehat{\mathbb{Z}}$ is called the minor arcs.

For groups other than $\mathbb{Z}$ in the present account I propose to replace arcs in corresponding definitions by cells.

Can we similarly decompose $\widehat{Q}_{1}, \widehat{Q}^{*}$ ? The decomposition of $\widehat{\mathbb{Z}}$ in the circle method varies according to the problem at hand. For $\widehat{Q}_{1}$ and problems involving shifted primes the following suggests itself.

Define a (translation invariant) metric $\varrho$ on $\widehat{Q}_{1}$ by

$$
\varrho(g, h)=\left(\sum_{\substack{p \leq N \\(p, N)=1}} \frac{1}{p}|g(p)-h(p)|^{2}\right)^{1 / 2} .
$$


For major cells we take the tubular neighbourhoods ("worms"):

$$
\left(g ; \inf _{|\tau| \leq T} \varrho\left(g, h_{\tau}\right) \leq \delta\right)
$$

where $h_{\tau}$ is the completely multiplicative function given by $h_{\tau}(q)=q^{i \tau} \chi(q)$ for a real $\tau$, and primitive Dirichlet character $\chi$. Strictly speaking a Dirichlet character $\chi(\bmod D)$ does not belong to $\widehat{Q}_{1}$ so, contrary to classical practice, we define $\chi$ to be 1 on the primes dividing $D$.

That $\chi$ be primitive corresponds to the restriction $(a, k)=1$ in the circle method. We would expect the order of $\chi$ (and the value of $T$ ) to be small compared to $N$. In a later section I show that under favourable circumstances these worms may be replaced by $\varrho$-spheres about (modified) Dirichlet characters.

What remains of $\widehat{Q}_{1}$ is called the minor cells.

I leave as a (not altogether easy) exercise to the reader that the (modified) Dirichlet characters are everywhere dense in $\widehat{Q}_{1}$. We shall not explicitly use this fact.

When studying $\widehat{Q^{*}}$, a family of metrics $\left(\sum p^{-\lambda}|g(p)-h(p)|^{2}\right)^{1 / 2}, \lambda>1$, seems appropriate.

Major arcs in the circle method. Attached to the major arc about the point $a k^{-1}(\bmod 1)$ is the asymptotic estimate

$$
\frac{1}{\pi(N)} \sum_{p \leq N} e^{2 \pi i a k^{-1} p} \rightarrow \frac{\mu(k)}{\phi(k)}, \quad N \rightarrow \infty,
$$

a result depending upon the distribution of primes in residue classes $(\bmod k)$. For a general $g_{\alpha}$ in this arc

$$
\frac{1}{\pi(N)}\left|\sum_{p \leq N} g_{\alpha}(p)\right| \approx \frac{|\mu(k)|}{\phi(k)} \min \left(1, \pi(N)^{-1} \sigma\left(g_{\alpha}, g_{a k^{-1}}\right)^{-1}\right),
$$

where $\approx$ denotes "behaves like".

Major cells in $\widehat{Q^{*}}$. It would appear that the multiplicative analogue of the prime $p$ is, for problems of prime pair type, the shifted prime $p+1$. For a primitive Dirichlet character $\chi(\bmod k)$,

$$
\frac{1}{\pi(N)} \sum_{p \leq N-1} \chi(p+1) \rightarrow \frac{\mu(k)}{\phi(k)}, \quad N \rightarrow \infty .
$$

The similarity with (1) is striking.

Major cells in $\widehat{Q}_{1}$. Attached to a worm about the (primitive) character $\chi(\bmod k)$ is the estimate

$$
\frac{1}{\pi(N)} \sum_{p \leq N} \chi(N-p) \rightarrow \frac{\mu(k) \chi(N)}{\phi(k)}, \quad N \rightarrow \infty .
$$


Generally

$$
\frac{1}{\pi(N)}\left|\sum_{p \leq N} g(N-p)\right| \approx \frac{|\mu(k) \chi(N)|}{\phi(k) \sqrt{1+\tau^{2}}} \exp \left(-\frac{1}{2} \varrho^{2}\left(g, h_{\tau}\right)\right) .
$$

Compared to (2), $|S(g)|$ peaks very much less violently, indeed it falls only slowly away from an extremum. As with the circle method, we might accelerate the process by considering powers $|S(g)|^{2 m}, m \geq 1$. This amounts to seeking a representation of the form

$$
2=\prod_{i=1}^{m}\left(N-p_{i}\right) \prod_{j=1}^{m}\left(N-q_{j}\right)^{-1}
$$

We might also replace $\widehat{Q}_{1}$ by $\left(\mathbb{C}^{*}\right)^{\pi(N)-\omega(N)}$, i.e. allow $g(p)=z_{p}$ complex and non-zero, and work in terms of many complex variables $z_{p}$.

Vinogradov effected his proof of Goldbach's conjecture for (sufficiently large) odd numbers by providing a non-trivial upper bound for $Y(\alpha)$ on the minor arcs.

A satisfactory bound for $S(g)$ on the minor cells of $\widehat{Q}_{1}$ is still wanting. To establish anything non-trivial at the moment we need not only that $g$ not lie in any (low-order worm) of the major cells, but that $g^{2}, g^{3}$ not lie there either. Since there are $3^{\pi(N)-\omega(N)}$ characters $g: Q_{1} \rightarrow U$ which satisfy $g^{3}=1$, there is at present a (corresponding) "third region" of $\widehat{Q}_{1}$ in which $g$ is between the major and the (reliably) minor cells.

In the following sections I show that something can still be done, although for the moment I abandon control on the number of factors in the representing product and aim at Conjecture III.

2. I give the notation again. Let $0<\delta \leq 1, N$ a positive integer, $P$ a set of primes not exceeding $N$ and coprime to $N$,

$$
|P|=\sum_{p \in P} 1 \geq \delta \pi(N)>0
$$

Let $Q_{1}$ be the multiplicative group generated by the positive integers $n$ not exceeding $N,(n, N)=1, \Gamma$ the subgroup of $Q_{1}$ generated by the $N-p$ with $p$ in $P, G_{1}$ the quotient group $Q_{1} / \Gamma$.

THEOREM 1. If $N \geq N_{0}(\delta)$, then we may remove a set of primes $q$, not exceeding $N$ and with $\sum q^{-1} \leq c_{1}(\delta)$, such that $G$, the subgroup of $G_{1}$ generated by the rationals in $Q_{1}$ with no q-factor, satisfies

(i) $|G| \leq c_{2}(\delta)$,

(ii) there is a subgroup $L$ of $G$ so that $G / L$ is arithmic $\left({ }^{1}\right)$,

(iii) $|L| \leq 4 / \delta$.

$\left({ }^{1}\right)$ The term "arithmic" is explained on the next page; see also [2], p. 392. 
Conjecture. In (iii) $4 / \delta$ should be $1 / \delta$. Then $\delta>1 / 2$ would force $|L|=1$, and $G$ itself would be arithmic. We may perhaps view (iii) as singular integral as geometric obstruction.

(ii) asserts the existence of a positive integer $D$ and a group homomorphism $(\mathbb{Z} / D \mathbb{Z})^{*} \rightarrow G / L$ which makes the following diagram commute:

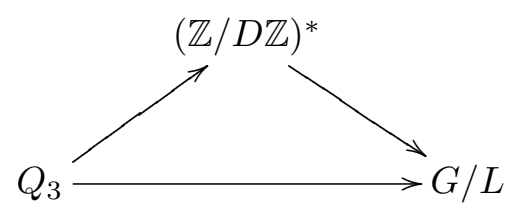

Here $Q_{3}$ is the subgroup of $Q_{1}$ when the $q$-factors are removed, $\left(D, Q_{3}\right)=$ $1,(\mathbb{Z} / D \mathbb{Z})^{*}$ is the multiplicative group of reduced residue classes $(\bmod D)$, the maps $Q_{3} \rightarrow(\mathbb{Z} / D \mathbb{Z})^{*}, Q_{3} \rightarrow G \rightarrow G / L$ are canonical. $D$ and the $c_{j}(\delta)$ may be effectively determined, but not the individual $q$. We may perhaps view (ii) as singular series. It asserts that the representability of an integer by products of the $N-p$ essentially depends upon the residue class $(\bmod D)$ to which it belongs.

Corollary. If $1 \leq r<s \leq N$, rs is coprime to $N$ and not divisible by $a q$, and if $r \equiv s(\bmod D)$, then there is a representation

$$
\left(\frac{r}{s}\right)^{|L|}=\prod_{p \in P}(N-p)^{d_{p}}
$$

with integer exponents $d_{p}$.

The proof of Theorem 1 is a little lengthy.

Lemma 1. Let $c>0$. If

$$
\sum_{\substack{q \leq N \\(q, M)=1 \\ q \text { prime }}} \frac{1}{q}\left(1-\operatorname{Re} q^{i \tau}\right) \leq \beta \leq \frac{1}{8} \log \log N
$$

where $|\tau| \leq N^{c}, 1 \leq M \leq N^{4}, N \geq e^{2}$, then

$$
\tau \log N \ll e^{\beta} .
$$

Proof. Without the condition $(q, M)=1$, a precise result of this type may be found in [3], Lemma 7.

We make three passes with our argument. Assume first that there is no condition $(q, M)=1$. Set $\sigma=1+(\log N)^{-1}$ and argue with Euler products:

$$
\left|\zeta(\sigma) \zeta(\sigma+i \tau)^{-1}\right|=\exp \left(\sum_{q \leq N} \frac{1}{q^{\sigma}}\left(1-\operatorname{Re} q^{i \tau}\right)+O(1)\right) \ll e^{\beta}
$$


Since $\zeta(\sigma+i \tau) \ll|\tau|^{-1}+(\log (2+|\tau|))^{3 / 4}$ (see [6], Théorème 11.1), and $(\sigma-1) \zeta(\sigma) \rightarrow 1$ as $N \rightarrow \infty$,

$$
\log N \ll\left(|\tau|^{-1}+(\log N)^{3 / 4}\right) e^{\beta} \ll|\tau|^{-1} e^{\beta}+(\log N)^{7 / 8} .
$$

This is the first pass.

We restore the condition $(q, M)=1$ and replace the use of $\zeta(s)$ by that of $\zeta(s) \prod_{q \mid M}\left(1-q^{-s}\right)$. This leads to a bound

$$
\log N \ll\left(\frac{M}{\phi(M)}\right)^{2} e^{\beta}\left(|\tau|^{-1}+(\log (2+|\tau|))^{3 / 4}\right) .
$$

Again the term involving $\log (2+|\tau|)$ may be omitted in favour of $\log N$. In particular, $\tau \ll(\log N)^{-7 / 8}(\log \log N)^{2}$. This is our second pass. It allows us to assert that

$$
\sum_{q \mid M} \frac{1}{q}\left(1-\operatorname{Re} q^{i \tau}\right) \ll \sum_{q \mid M} \frac{|\tau| \log q}{q} \ll|\tau| \log \log M \ll(\log N)^{-1 / 2} .
$$

Note that for any $y \geq 2$,

$$
\sum_{q \mid M} \frac{\log q}{q} \ll \sum_{q \leq y} \frac{\log q}{q}+\frac{\log y}{y} \sum_{\substack{q \mid M \\ q>y}} 1 \ll \log y+\frac{\log y}{y} \cdot \frac{\log M}{\log y},
$$

and we may set $y=\log M$.

At the expense of replacing $\beta$ by $\beta+O\left((\log N)^{-1 / 2}\right)$ we may remove the condition $(q, M)=1$ from the hypothesis of the lemma and proceed as initially. This is the third pass.

LEMMA 2. Let $g_{j}, 1 \leq j \leq k$, be multiplicative functions with values in the complex unit disc. The inequality

$$
\sum_{p<N}\left|\sum_{j=1}^{k} c_{j} g_{j}(N-p)\right|^{2} \leq \lambda \sum_{j=1}^{k}\left|c_{j}\right|^{2},
$$

with

$$
\begin{aligned}
\lambda= & 4 \pi(N)+\frac{\gamma_{0} N}{\phi(N) \log N} \max _{1 \leq j \leq k} \max _{\chi(\bmod d)} \frac{d}{\phi(d)^{2}} \sum_{\substack{l=1 \\
l \neq j}}^{k}\left|\sum_{\substack{n<N \\
(n, N)=1}} g_{j}(n) \overline{g_{l}(n)} \chi(n)\right| \\
& +O\left(N(\log N)^{-21 / 20}\right)
\end{aligned}
$$

is valid for all complex $c_{j}$ and all $N \geq e^{2}$. Here $\gamma_{0}$ is absolute and the innermost maximum runs over the Dirichlet characters to squarefree moduli $d$.

Proof. This is an analogue of Theorem 3 of [5], and may be obtained in the same way. No doubt a result of this type holds with 1 in place of the leading coefficient 4 . 
LEMma 3. There is a positive $c$ so that

$$
\phi(N)^{-1} \sum_{\substack{n \leq N \\(n, N)=1}} g(n) \ll T^{-c}+\exp \left(-c \min _{|\tau| \leq T} \sum_{\substack{q \leq N \\(q, N)=1 \\ q \text { prime }}} \frac{1}{q}\left(1-\operatorname{Re} g(q) q^{i \tau}\right)\right)
$$

uniformly for multiplicative $g$ with values in the complex unit disc, $T \geq 1$, $N \geq e^{2}$.

P r o o f. The classical treatment of Halász, [7], needs a modification, such as that carried out in [4], Lemma 12.

3. Proof of Theorem 1, first step. Let $U$ be the complex unit disc. Until further notice $\chi$ will revert to its classical meaning.

LEMMA 4. If $g: Q_{1} \rightarrow Q_{1} / \Gamma \rightarrow U$ extends a character on $G_{1}$, then there is an integer $m, 1 \leq m \leq 4 / \delta$, a Dirichlet character $\chi$ to a squarefree modulus $d$ not exceeding a bound depending only upon $\delta$, and a constant $\gamma$, also depending at most upon $\delta$, so that

$$
\sum_{\substack{q \leq N,(q, N)=1 \\ \chi(q) g(q)^{m} \neq 1}} \frac{1}{q} \leq \gamma
$$

REMARKs. The exceptional set of primes $q$ may vary with $g$. The bound $4 / \delta$ should no doubt be $\delta^{-1}$.

Pr o of (of Lemma 4). We obtain upper and lower bounds for

$$
S=\sum_{j=1}^{k}\left|\sum_{p \in P}(g(N-p))^{j}\right|^{2}
$$

where the $N-p$ belong to the set of integers generating $\Gamma$.

A lower bound is $k(\delta \pi(N))^{2}$.

The inequality dual to that in Lemma 2 asserts that

$$
\sum_{j=1}^{k}\left|\sum_{p \leq N} a_{p} g_{j}(N-p)\right|^{2} \leq \lambda \sum_{p \leq N}\left|a_{p}\right|^{2}
$$

for all complex $a_{p}$. Setting $g_{j}(n)=(g(n))^{j}$ and choosing the $a_{p}$ appropriately gives an upper bound $S \leq|P| \lambda$. Combined with the lower bound this yields

$$
\begin{aligned}
k \delta \leq & +\gamma_{1} \max _{\chi(\bmod d)} \frac{d}{\phi(d)^{2}} \sum_{j=1}^{k-1} \frac{1}{\phi(N)}\left|\sum_{\substack{n<N \\
(n, N)=1}} g(n)^{j} \chi(n)\right| \\
& +O\left((\log N)^{-1 / 20}\right)
\end{aligned}
$$

for an absolute constant $\gamma_{1}$. 
Let $0<3 \varepsilon<\delta$. Replacing $\delta$ by $\delta-\varepsilon$ and fixing $d_{0}$ at a sufficiently large value in terms of $\varepsilon$ allows us to confine the maximum to the range $1 \leq d \leq d_{0}$ (still over squarefree moduli).

We estimate the innermost sum of (3) by Lemma 3. Fixing $T$ at a value sufficiently large in terms of $\varepsilon$ shows that

$k(\delta-2 \varepsilon)$

$$
\begin{aligned}
\leq & +\gamma_{2} \sum_{j=1}^{k} \exp \left(-c \min _{|\tau| \leq T} \min _{\chi(\bmod d)} \sum_{\substack{q \leq N \\
(q, N)=1 \\
q \text { prime }}} \frac{1}{q}\left(1-\operatorname{Re} g(q)^{j} \chi(q) q^{i \tau}\right)\right) \\
& +O\left((\log N)^{-1 / 20}\right) .
\end{aligned}
$$

Again $\gamma_{2}$ is absolute.

This inequality holds for all positive integers $k$.

Denote the double minimum by $m_{j}\left(=m_{j}(T)\right)$. Let $B$ denote the sequence of positive integers $j$ for which $m_{j} \leq M$. This is not the $M$ of Lemma 1. Here

$$
k\left(\delta-2 \varepsilon-\gamma_{2} \exp (-c M)\right) \leq 4+O\left((\log N)^{-1 / 20}\right)+\gamma_{2} \sum_{\substack{j=1 \\ m_{j} \leq M}}^{k} 1 .
$$

Fixing $M$ large enough in terms of $\varepsilon$ we see that the sequence $B$ has a lower asymptotic density of at least $\delta-3 \varepsilon$. Let $r$ be the highest common factor of the integers in $B$. By adjoining 1 to $B$ and using Schnirelmann's addition theorems (cf. [1], Chapter 8; [2], Chapter 22), we see that every sufficiently large integer $t$ has a representation $r t=j_{1}+\ldots+j_{s}$, with $r, s$ bounded in terms of $\delta-3 \varepsilon$.

Since

$$
1-\operatorname{Re} z_{1} \ldots z_{w} \leq \sum_{u=1}^{w} w\left(1-\operatorname{Re} z_{u}\right)
$$

for $z_{u}$ in the unit disc,

$$
m_{r t}(s T)=m_{j_{1}+\ldots+j_{s}}(s T) \leq s \sum_{u=1}^{s} m_{j_{u}}(T) \leq s M .
$$

The inequality

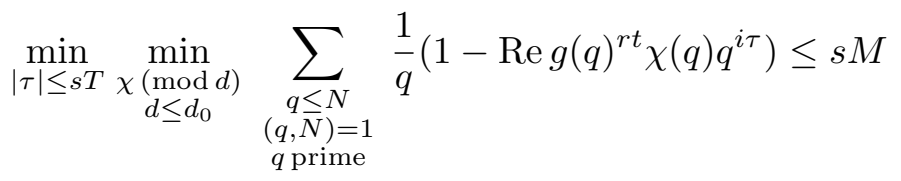

holds for all positive integers $t$. 
There is an integer $v$, not exceeding $\left[d_{0}\right]$ !, for which every $\chi^{v}$ is principal. Replacing $r t, \tau, s$ by $r t v, \tau v, v^{2} s$ respectively, we may remove the character $\chi(q)$ from the last inequality. In particular

$$
\sum_{\substack{q \leq N \\(q, N)=1 \\ q \text { prime }}} \frac{1}{q}\left(1-\operatorname{Re} g(q)^{v r t} q^{i \tau(t)}\right) \leq v^{2} s M+v
$$

for a certain $\tau(t)$, not exceeding $v s T$ in absolute value, and so bounded in terms of $\delta, \varepsilon$. that

Since $g(q)^{v r t_{1}} g(q)^{v r t_{2}} \overline{g(q)} \operatorname{vr}\left(t_{1}+t_{2}\right)=1$, we can further argue from (4)

$$
\sum_{\substack{q \leq N \\(q, N)=1 \\ q \text { prime }}} \frac{1}{q}\left(1-\operatorname{Re} q^{i v\left(\tau\left(t_{1}\right)+\tau\left(t_{2}\right)-\tau\left(t_{1}+t_{2}\right)\right)}\right) \leq 3 v(v s M+1),
$$

uniformly for all positive integers $t_{j}$. We are ready to apply Lemma 1 , and conclude that for $N$ sufficiently large in terms of $\delta, \varepsilon$,

$$
\tau\left(t_{1}\right)+\tau\left(t_{2}\right)-\tau\left(t_{1}+t_{2}\right) \ll(\log N)^{-1},
$$

uniformly in the $t_{j}$.

There is now an $\omega$ such that $\tau(t)-t \omega \ll(\log N)^{-1}$ for all positive $t$. This particular result goes back to Exercise 99 (Chapter 3, p. 17) of Pólya and Szegö, [8]. However, in our case the sequence $\tau(t)$ is uniformly bounded in terms of $\delta, \varepsilon$. Thus $\omega$ must be zero, $\tau(T) \ll(\log N)^{-1}$ uniformly in $t$.

We return to the inequality (5) and remove the $\tau(t)$ :

$$
\sum_{\substack{q \leq N \\(q, N)=1 \\ q \text { prime }}} \frac{1}{q}\left(1-\operatorname{Re} g(q)^{v r t}\right) \ll 1,
$$

since

$$
\sum_{q \leq N} \frac{\left|q^{i \tau(t)}-1\right|}{q} \ll|\tau(t)| \sum_{q \leq N} \frac{\log q}{q} \ll 1, \quad t=1,2, \ldots
$$

We are nearly there. For $|\theta| \leq 1$,

$$
\lim _{k \rightarrow \infty} \frac{1}{k} \sum_{t=1}^{k} \theta^{t}= \begin{cases}1 & \text { if } \theta=1 \\ 0 & \text { else. }\end{cases}
$$

The uniformity of our inequality (7) then shows that

$$
\sum_{\substack{q \leq N,(q, N)=1 \\ g(q)^{v r} \neq 1}} \frac{1}{q} \ll 1
$$


the upper bound depending only upon $\delta, \varepsilon$. This is the asserted result save that $v r$ is not explicitly bounded in terms of $\delta$.

Looking back to (3), near the beginning of this lemma, with $k$ chosen so that $k \delta>4$ we can find an integer $j, 1 \leq j \leq k-1$, for which

$$
\frac{1}{\phi(N)}\left|\sum_{\substack{n<N \\(n, N)=1}} g(n)^{j} \chi(n)\right| \geq y_{1}(\delta, k)>0 .
$$

With $T, d_{1}$ sufficiently large in terms of $y_{1}$,

$$
\exp \left(-c \min _{d \leq d_{1}} \min _{|\tau| \leq T} \sum_{\substack{q \leq N \\(q, N)=1}} \frac{1}{q}\left(1-\operatorname{Re} g(q)^{j} \chi(q) q^{i \tau}\right)\right)>y_{2}>0 .
$$

For some $d$ not exceeding $d_{1},|\tau| \leq T$,

$$
\sum_{\substack{q \leq N \\(q, N)=1}} \frac{1}{q}\left(1-\operatorname{Re} g(q)^{j} \chi(q) q^{i \tau}\right) \leq y_{3}(\delta, k) .
$$

By adjusting $y_{3}$ upwards if necessary, we can adjoin the condition $g(q)^{v r}=1$ to the sum. Raising $g(q)^{j} \chi(q) q^{i \tau}$ to its $v r$ th power, we see that $\tau \log N \ll 1$. Again we may remove $\tau$ :

$$
\sum_{\substack{q \leq N \\(q, N)=1}} \frac{1}{q}\left(1-\operatorname{Re} g(q)^{j} \chi(q)\right) \leq y_{4}(\delta, k) .
$$

If $g(q)^{j} \chi(q)$ is not 1 , then since it is a vrth root of unity,

$$
1-\operatorname{Re} g(q)^{j} \chi(q) \geq \min _{\substack{(a, b)=1 \\ 2 \leq b \leq v r}}\left(1-\operatorname{Re} \exp \left(2 \pi i a b^{-1}\right)\right) \geq y_{5}>0 .
$$

Thus

$$
\sum_{\substack{q \leq N,(q, N)=1 \\ g(q)^{j} \chi(q) \neq 1}} \frac{1}{q} \leq y_{6}(\delta, k)
$$

We can choose any $k>4 \delta^{-1} ; k=\left[4 \delta^{-1}\right]+1$ will do.

The proof was constructed assuming $N$ to be sufficiently large in terms of $\delta$. For the finitely many remaining values of $N$ Lemma 4 is trivially valid.

4. Proof of Theorem 1, second step. We set out to make the exceptional set of primes $q$ in Lemma 4 uniform in $g$. The notation of the previous section remains in force.

Lemma 5. There is a subgroup $G_{2}$ of $Q_{1} / \Gamma$ with the property that the primes $q$ taken by the canonical map $Q_{1} \rightarrow Q_{1} / \Gamma$ onto any of the cosets 
outside of $G_{2}$, have the sum of their reciprocals bounded independently of $N$. Moreover, the order of $G_{2}$ does not exceed a value depending only upon $\delta$.

REMARK. In particular, we may delete the character in Lemma 4, and choose a common value for the powers $m$, uniform in $g$.

Let $h$ denote a typical character on $G_{1}=Q_{1} / \Gamma$, and $g$ its extension to $Q_{1}$ :

$$
g: Q_{1} \rightarrow G_{1} \stackrel{h}{\rightarrow} U
$$

If $t_{1}, \ldots, t_{s}$ are distinct elements of $G_{1}$, and $p \mapsto \bar{p}$ denotes the action of the canonical map $Q_{1} \rightarrow G_{1}$, then

$$
\sum_{\substack{p<N \\(p, N)=1}} \frac{1}{p}(1-\operatorname{Re} g(p) \chi(p)) \geq \sum_{j} \sum_{\omega}\left(1-\operatorname{Re} h\left(t_{j}\right) \omega\right) \beta_{j, \omega}=L(h, \chi),
$$

say, where $\omega$ runs through the values assumed by $\chi$, and $\beta_{j, \omega}$ is any real non-negative number not exceeding

$$
\sum_{\substack{p<N,(p, N)=1 \\ \bar{p}=t_{j}, \chi(p)=\omega}} \frac{1}{p}
$$

It will be convenient to choose for $\beta_{j, \omega}$ the minimum of this sum and $\alpha$, with $\alpha$ to be fixed later. For ease of presentation set $\beta_{j}=\sum_{\omega} \beta_{j, \omega}$. Thus

$$
0 \leq \beta_{j} \leq \sum_{\substack{p<N,(p, N)=1 \\ \bar{p}=t_{j}}} p^{-1}
$$

In terms of the metric $\varrho(g, h)$ defined on $\widehat{Q}_{1}$ in Section 1 , we have

$$
\frac{1}{2} \varrho(g, h)^{2}=\sum_{\substack{p<N \\(p, N)=1}} \frac{1}{p}(1-\operatorname{Re} g(p) \overline{h(p)}) .
$$

For $s$ large enough $L(h, \chi)$ may be considered essentially $\frac{1}{2} \varrho(g, \chi)^{2}$. We extend $\varrho$ to a metric on $\mathbb{C}^{\pi(N)-\omega(N)}$ and regard $\widehat{Q}_{1}$ for topological purposes as a subset of $\mathbb{C}^{\pi(N)-\omega(N)}$. This loses us the translation invariance of $\varrho$ on $\widehat{Q}_{1}$ but allows the choice of a standard Dirichlet character for $g, h$.

We wish to estimate how often the distances $\varrho\left(g_{i}, g_{j} \chi\right)$ can be small, for $1 \leq i<j \leq v$, and all (standard) $\chi$ to moduli not exceeding $d_{0}$, say. We move this question onto $\widehat{G}_{1}$.

Let $\mu$ be the Haar measure on $\widehat{G}_{1}$, normalised so that $\mu \widehat{G}_{1}=1$.

LEMMA 6.

$$
\mu\left(h \in \widehat{G}_{1} ; L(h, \chi) \leq \frac{1}{2} \sum_{j=1}^{s} \beta_{j}\right) \leq 4\left(\sum_{j=1}^{s} \beta_{j}\right)^{-2} \sum_{j}\left|\sum_{\omega} \omega \beta_{j, \omega}\right|^{2} .
$$


Proof. Arguing as Chebyshev would, the desired measure does not exceed

$$
\begin{aligned}
& \mu\left(h \in \widehat{G}_{1} ; \operatorname{Re} \sum_{j=1}^{s}\right.\left.\sum_{\omega} \omega \beta_{j, \omega} h\left(t_{j}\right) \geq \frac{1}{2} \sum_{j=1}^{s} \beta_{j}\right) \\
& \leq \mu\left(h \in \widehat{G}_{1} ;\left|\sum_{j=1}^{s} \sum_{\omega} \omega \beta_{j, \omega} h\left(t_{j}\right)\right| \geq \frac{1}{2} \sum_{j=1}^{s} \beta_{j}\right) \\
& \leq 4\left(\sum_{j=1}^{s} \beta_{j}\right)^{-2} \int_{h \in \widehat{G}_{1}}\left|\sum_{j=1}^{s}\left(\sum_{\omega} \omega \beta_{j, \omega}\right) h\left(t_{j}\right)\right|^{2} d \mu(h) \\
&=4\left(\sum_{j=1}^{s} \beta_{j}\right)^{-2} \sum_{j=1}^{s}\left|\sum_{\omega} \omega \beta_{j, \omega}\right|^{2} .
\end{aligned}
$$

Let $\theta(\chi)$ denote the upper bound in Lemma 6, and set

$$
\theta=\sum_{d \leq d_{0}} \sum_{\chi(\bmod d)} \theta(\chi)
$$

the moduli $d$ assumed squarefree.

LEMmA 7 (Well-spaced functions on $\widehat{G}_{1}$ ). If $v^{2} \theta<1$, then there are functions $h_{j}, 1 \leq j \leq v$, in $\widehat{G}_{1}$, such that

$$
L\left(h_{i} \bar{h}_{k}, \chi\right) \geq \frac{1}{2} \sum_{j=1}^{s} \beta_{j} \quad \text { for } 1 \leq i<k \leq v,
$$

for every $\chi(\bmod d), d \leq d_{0}$.

Proof. Any character on $G_{1}$ will serve for $h_{1}$. Using the translation invariance of Haar measure, the previous lemma guarantees that

$$
\mu\left(h \in \widehat{G}_{1} ; L\left(h_{1} \bar{h}, \chi\right) \leq \frac{1}{2} \sum_{j=1}^{s} \beta_{j} \text { for some } \chi(\bmod d), d \leq d_{0}\right)
$$

does not exceed $\theta$. There is an $h$ for which $L\left(h_{1} \bar{h}, \chi\right)$ is suitably large.

We successively remove sets to obtain functions $h_{i}$ inductively. Having $h_{i}, 1 \leq i \leq k-1$, an $h_{k}$ may be chosen, so that every $L\left(h_{i} \bar{h}_{k}, \chi\right)$ is suitably large, by removing from $\widehat{G}_{1}$ a set of $\mu$-measure at most $(k-1) \theta$.

Since $\theta(1+2+\ldots+v-1)=\theta \frac{1}{2}(v-1) v \leq v^{2} \theta<1, v$ steps of this argument are possible.

We return to the group $\widehat{Q}_{1}$.

Lemma 8 (In a worm is in a sphere). Suppose $T \leq N, \chi_{1}, \chi_{2}$ are Dirichlet characters of order $\leq b \leq N$, to moduli $\leq N$, and $m$ is a positive integer 
not exceeding $N$. Then

$$
\varrho\left(g, \chi_{1}\right) \ll \exp \left(\sqrt{m b} \min _{|\tau| \leq T} \varrho\left(g, \chi_{1} p^{i \tau}\right)+\sqrt{b} \varrho\left(g^{m}, \chi_{2}\right)\right) .
$$

Proof. Choose $\tau$ to minimize $\varrho\left(g, \chi p^{i \tau}\right)$ subject to $|\tau| \leq T$ (the completely multiplicative function $\chi p^{i \tau}$ has value $\chi(p) p^{i \tau}$ on the prime(s) $p$ ), and let $\delta$ denote the minimum value. By (4), with the $z_{j}$ equal,

$$
\varrho\left(g^{m}, \chi_{1}^{m} p^{i m \tau}\right) \leq m^{1 / 2} \varrho\left(g, \chi_{1} p^{i \tau}\right)=m^{1 / 2} \delta .
$$

By the triangle inequality ( $\varrho$ viewed on $\left.\mathbb{C}^{\pi(N)-\omega(N)}\right)$,

$$
\varrho\left(\chi_{2}, \chi_{1}^{m} p^{i m \tau}\right) \leq m^{1 / 2} \delta+\varrho\left(g^{m}, \chi_{2}\right) .
$$

If $\bar{\chi}_{2} \chi_{1}$ is defined $(\bmod w)$, then

$$
\left(\sum_{\substack{p<N \\(p, N w)=1}} \frac{1}{p}\left|1-\bar{\chi}_{2} \chi_{1}^{m}(p) p^{i m \tau}\right|^{2}\right)^{1 / 2}
$$

falls under the same bound. Let $\bar{\chi}_{2} \chi_{1}^{m}$ have order $\Delta$. Then again by (4),

$$
\left(\sum_{\substack{p<N \\(p, N w)=1}} \frac{1}{p}\left|1-p^{i m \tau} \Delta\right|^{2}\right)^{1 / 2} \leq \Delta^{1 / 2}\left(m^{1 / 2} \delta+\varrho\left(g^{m}, \chi_{2}\right)\right) .
$$

Note that $w \leq N^{2}, \Delta \leq b^{2}$. We may therefore appeal to Lemma 1 of Section 2 and deduce that

$$
\begin{aligned}
m \tau \Delta \log N & \ll \exp \left(\Delta^{1 / 2}\left(m^{1 / 2} \delta+\varrho\left(g^{m}, \chi_{2}\right)\right)\right) \\
& \ll \exp \left((b m)^{1 / 2} \delta+b^{1 / 2} \varrho\left(g^{m}, \chi_{2}\right)\right),
\end{aligned}
$$

provided the final exponent does not exceed $\frac{1}{8} \log \log N$.

Again by the triangle inequality

$$
\varrho\left(g, \chi_{1}\right) \leq \varrho\left(g, \chi_{1} p^{i \tau}\right)+\varrho\left(\chi_{1} p^{i \tau}, \chi_{1}\right)
$$

The second of the bounding terms is

$$
\ll\left(\sum_{p<N} \frac{1}{p}\left|p^{i \tau}-1\right|^{2}\right)^{1 / 2} \ll\left(|\tau|^{2} \sum_{p<N} \frac{(\log p)^{2}}{p}\right)^{1 / 2} \ll|\tau| \log N,
$$

and the inequality of the lemma follows readily.

Otherwise $(b m)^{1 / 2} \delta+b^{1 / 2} \varrho\left(g^{M}, \chi_{2}\right)>\frac{1}{8} \log \log N$ and the asserted inequality of Lemma 8 is "trivially" valid.

REMARK. According to Lemma 4, for each (extended) character $g$ on $Q_{1}$, there is an $m, 1 \leq m \leq 4 / \delta$, and a Dirichlet character $\chi_{2}$ to a modulus not exceeding a function of $\delta$, so that $\varrho\left(g^{m}, \chi_{2}\right) \ll 1$, uniformly in $g, N$. Since the number of possible $\chi_{2}$ is bounded in terms of $\delta$, we may take the same 
value of $m$ for all the $\chi_{2}$. With this value of $m$, Lemma 8 shows that for any $\chi_{1}$ to a modulus not exceeding $N$, and of order at most $b$,

$$
\varrho\left(g, \chi_{1}\right) \ll \exp \left((m b)^{1 / 2} \min _{|\tau| \leq N} \varrho\left(g, \chi_{1} p^{i \tau}\right)\right) .
$$

This explains the subtitle of Lemma 8 .

We put the results of these last two subsections together. Let $t_{1}, \ldots, t_{s}$ be elements in $G_{1}$. Suppose that $\theta<1$, and let $v=\left[\theta^{-1 / 2}\right]$. If $\theta=0$, then we can choose any positive value for $v$. Thus $v \geq 1$.

Let $h_{1}, \ldots, h_{v}$ be functions in $\widehat{G}_{1}$, guaranteed by Lemma 7 , for which the $L\left(h_{i} \bar{h}_{k}, \chi\right)$ are large.

Extend the $h_{i}$ to $g_{i}$ on $Q_{1}$. Then

$$
\begin{aligned}
\exp \left(\left(m d_{0}\right)^{1 / 2} \min _{|\tau| \leq N} \sum_{\substack{p<N \\
(p, N)=1}} \frac{1}{p}\left(1-\operatorname{Re} g_{i} \bar{g}_{k} \chi_{1}(p) p^{i \tau}\right)\right) \\
\gg \sum_{\substack{p<N \\
(p, N)=1}} \frac{1}{p}\left(1-\operatorname{Re} g_{i} \bar{g}_{k} \chi_{1}(p)\right) \\
\gg L\left(h_{i} \bar{h}_{k}, \chi_{1}\right) \gg \sum_{j=1}^{s} \beta_{j}, \quad 1 \leq i<k \leq v,
\end{aligned}
$$

for all Dirichlet characters $\chi_{1}$ to moduli at most $d_{0}(\leq N)$.

Appeal to Lemma 3 shows that for a certain positive (absolute) constant $c$,

$$
\phi(N)^{-1} \sum_{\substack{n<N \\(n, N)=1}} g_{i} \bar{g}_{k} \chi_{1}(n) \ll N^{-c}+\left(\sum_{j=1}^{s} \beta_{j}\right)^{-c\left(m d_{0}\right)^{-1 / 2}}
$$

uniformly for $1 \leq i<k \leq v$ and all $\chi_{1}$ to moduli not exceeding $d_{0}$.

We render the exceptional set in Lemma 4 effectively uniform in $g$ by estimating

$$
\sum_{j=1}^{v}\left|\sum_{p \in P} g_{j}(N-p)\right|^{2}
$$

from above and below. Again we appeal to the inequality dual to that of Lemma 2. This time

$$
\begin{aligned}
\delta v \leq 4 & +O\left(N^{-c}+\max _{d \leq d_{0}} \max _{(\bmod d)}\left(\sum_{j=1}^{s} \beta_{j}\right)^{-c\left(m d_{0}\right)^{-1 / 2}}\right) \\
& +O\left(d_{0}^{-1 / 2}\right)+O\left((\log N)^{-1 / 20}\right) .
\end{aligned}
$$


If $d_{0}$ is fixed at a value sufficiently large in terms of $\delta$, and $\theta$ does not exceed a certain value $\theta_{0}$, depending only upon $\delta$, then the terms 4 and $O\left(d_{0}^{-1 / 2}\right)$ will together not exceed $\delta v / 4$. If $N$ is large enough in terms of $\delta$, then for some $\chi_{1}$ to a modulus not exceeding $d_{0}, \sum_{j=1}^{s} \beta_{j}$ will be bounded in terms of $\delta$ alone.

However, $\theta>\theta_{0}$ entails

$$
\left(\sum_{j=1}^{s} \beta_{j}\right)^{2}<4 \theta_{0}^{-1} d_{0}^{2} \sum_{j=1}^{s}\left|\sum_{\omega} \omega \beta_{j, \omega}\right|^{2}
$$

for some character $\chi(\bmod d), d \leq d_{0}$. Here $|\omega| \leq 1, \beta_{j, \omega} \leq \alpha$, so that the upper bound does not exceed $r \theta_{0}^{-1} d_{0}^{2} \alpha \sum_{j=1}^{s} \beta_{j}$. With $\alpha=\theta_{0}\left(4 d_{0}^{2}\right)^{-1}$, $\sum_{j=1}^{s} \beta_{j} \leq 1$ ensues.

In either case $\sum_{j=1}^{s} \beta_{j}$ is bounded in terms of $\delta$ alone, i.e.

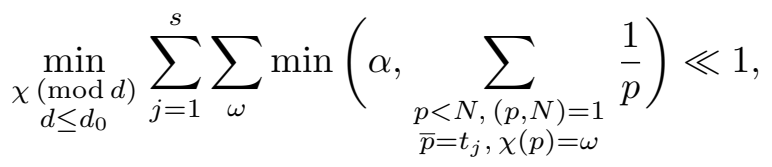

uniformly in $s, N$.

In our present circumstances we may allow the $t_{i}$ to run through all the elements of $G_{1}$. Those for which the innermost minimum is $\alpha$ are bounded in number in terms of $\delta$ alone. They generate a subgroup $G_{2}$ of $G_{1}$ of order bounded in terms of $\delta$.

For the remaining elements of $G_{1}$, which without loss of generality we again enumerate by $t_{j}, j=1,2, \ldots$, we see that

$$
\sum_{j=1}^{\infty} \sum_{\substack{p<N,(p, N)=1 \\ \bar{p}=t_{j}}} \frac{1}{p} \ll 1 .
$$

We have reached the following situation:

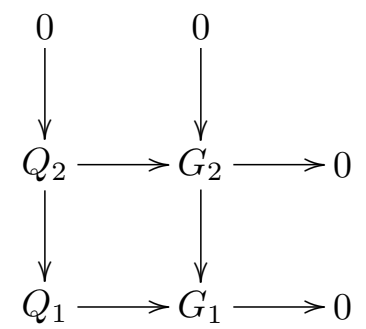

where the vertical maps denote identification, and $Q_{2}$ is derived from $Q_{1}$ by stripping a set of primes $q$ for which $\sum q^{-1}$ is bounded in terms of $\delta$ alone. We have shown that $\left|G_{2}\right| \leq c_{0}(\delta)$ uniformly in $N$.

This establishes Lemma 5 and part of Theorem 1. 
5. Proof of Theorem 1, third step. Arithmicity. We modify the above argument, with $t_{1}, \ldots, t_{s}$ running through the elements of $G_{2}$, and characters $h: G_{2} \rightarrow U$ extended canonically and then by projection to $g: Q_{1} \rightarrow Q_{2} \rightarrow G_{2} \rightarrow U$. Thus $g(q)=1$ on a set of primes $q$ for which $\sum q^{-1}$ converges.

Let $H$ be the subgroup of $\widehat{G}_{2}$ generated by characters $h$ that extend to a $g$ such that for some Dirichlet character $\chi$, to a modulus not exceeding $d_{1}$, $\sum p^{-1}$ taken over the primes $p<N, p \mid Q_{2}, g(p) \chi(p) \neq 1$, does not exceed $c_{1}$.

Lemma 9. If $d_{1}, c_{1}$ are fixed at sufficiently large values, depending at most upon $\delta$, then $\left|\widehat{G}_{2} / H\right| \leq 4 / \delta$.

Replacing 4 by 1 in Lemma 2 would replace 4 by 1 here.

Proof. If $h_{1}, h_{2}$ in $\widehat{G}_{2}$ belong to distinct cosets of $H$, then the corresponding extensions $g_{j}: Q_{1} \rightarrow Q_{2} \rightarrow G_{2} \rightarrow U, j=1,2$, satisfy

$$
\sum_{\substack{p<N \\ p \mid Q_{2}}} \frac{1}{p}\left(1-\operatorname{Re} g_{1} \bar{g}_{2} \chi(p)\right)>c_{1}
$$

for all $\chi(\bmod d), d \leq d_{1}$. Supposing we can find $s$ distinct such coset representatives, then the corresponding $s$ extensions $g_{j}$ satisfy

$$
\begin{aligned}
s|P|^{2} & =\sum_{j=1}^{s}\left|\sum_{p \in P} g_{j}(N-p)\right|^{2} \\
& \leq\left(4+O\left((\log N)^{-1 / 20}+c_{1}^{-1 / m d_{1}}\right)+O\left(d_{1}^{-1 / 2}\right)\right) \pi(N)|P|,
\end{aligned}
$$

where $m$ may be taken to be the same value as earlier provided $c_{1}$ is fixed large enough. If $d_{1}, c_{1}, N$ are sufficiently large (in terms of $\delta$ ), then $s \leq[4 / \delta]$. Here we use the fact that $s$ is an integer. This establishes the lemma.

Let $J$ be the subgroup of $G_{2}$ on which $H$ is trivial.

We remove from $Q_{2}$ all primes $p_{1}$ counted in a sum $\sum p_{1}^{-1}, p_{1}<N$, $p \mid Q_{2}, g \chi\left(p_{1}\right) \neq 1$, for some $g$ induced from $H$. These satisfy

$$
\omega_{0} \sum \frac{1}{p_{1}} \leq\left|\widehat{G}_{2}\right| c_{1}=\left|G_{2}\right| c_{1} \leq c_{3}(\delta)<\infty,
$$

where

$$
\omega_{0}=\min _{g \chi(p) \neq 1}(1-g \chi(p)) .
$$

Note that $g$ is defined on $G_{2}$, so satisfies $g(p)^{\left|G_{2}\right|}=1$. Since the modulus of $\chi$ does not exceed $d_{1}, \chi^{r}$ is principal for some $r$ not exceeding the least common multiple of the integers up to $\left[d_{1}\right]$. Thus once $\delta$ is fixed, $g \chi(p)$ 
belongs to a fixed set of roots of unity. An explicit lower bound can be given for $\omega_{0}$, depending upon $\delta$ alone.

It is convenient to also remove from $Q_{2}$ the primes not exceeding $d_{1}$. Call the resulting subgroup of $Q_{2}, Q_{3}$. Let $G_{3}$ be the subgroup of $G_{2}$ that it generates $(\bmod \Gamma)$.

We reach

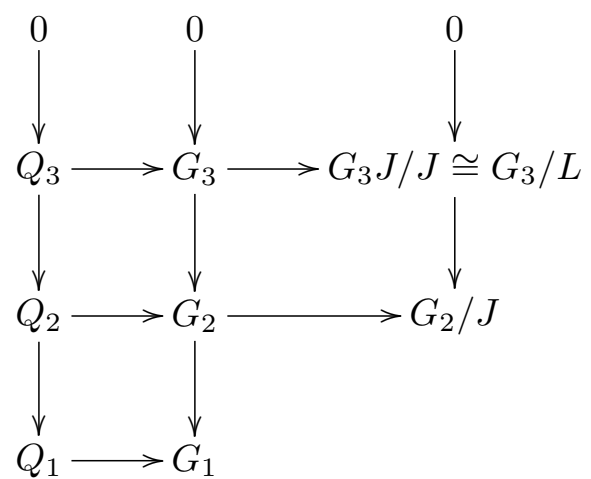

In this diagram $G_{j}=Q_{j} \Gamma / \Gamma, j=2,3$. By standard theorems in group theory, $G_{3} J / J \simeq G_{3} / G_{3} \cap J=G_{3} / L$, say. Note that $G_{3} / L$ may be viewed as a subgroup of $G_{2} / J$. In particular, $|L|=\left|G_{3} \cap J\right| \leq|J|$.

We have defined $J$ so that the upper exact sequence

$$
\begin{aligned}
& 0 \longleftarrow \widehat{G}_{2} / H \longleftarrow \widehat{G}_{2} \longleftarrow H \longleftarrow 0 \\
& 0 \longrightarrow G_{2} \longrightarrow G_{2} / J \longrightarrow 0
\end{aligned}
$$

is dual to the lower exact sequence, term by term. Therefore $|J|=|\widehat{J}|=$ $\left|\widehat{G}_{2} / H\right| \leq 4 / \delta$. Hence $|L| \leq 4 / \delta$.

We prove that $G_{3} / L$ is arithmic.

Let $h$ be a character on $G_{3} / L$. Since $U$ is $\mathbb{Z}$-divisible, there is a character $h^{\prime}: G_{2} / J \rightarrow U$ which coincides with $h$ on $G_{3} / L$. Here we use the identification of $G_{3} / L$ as a subgroup of $G_{2} / J$. We then lift $h^{\prime}$ up to $Q_{1}$ in the natural way:

$$
g: Q_{1} \rightarrow Q_{2} \rightarrow G_{2} \rightarrow G_{2} / J \stackrel{h^{\prime}}{\rightarrow} U .
$$

Since $h^{\prime}$ belongs to $\left(G_{2} / J\right)^{\wedge}$, i.e. to $H$, we may view $g$ as "induced from $H$ ".

Attached to $g$ there is a Dirichlet character $\chi$, to a modulus not exceeding $d_{1}$, so that $g$ coincides with $\chi$ on $Q_{3}$. Let $D$ be the product of the primes not exceeding $d_{1}$. In the previous statement we may replace $\chi$ by the character it induces mod $D$. (Remember that the $\chi$ have squarefree moduli, although the argument could be adjusted if they did not.)

Let $\sigma$ denote the composition of canonical maps $Q_{3} \rightarrow G_{3} \rightarrow G_{3} / L$. 
For integers $a, b$ dividing $Q_{3}$, and satisfying $a \equiv b(\bmod D)$, the lifting $g$ satisfies $g(a) \overline{g(b)}=\chi(a) \overline{\chi(b)}=1$. Otherwise expressed, $h(\sigma(a) / \sigma(b))=$ $h(\sigma(a)) \overline{h(\sigma(b)})=1$. Since this holds for all characters $h$ on $G_{3} / L, \sigma(a) / \sigma(b)$ is the identity of $G_{3} / L$. The map $a(\bmod D) \rightarrow \sigma(a)$

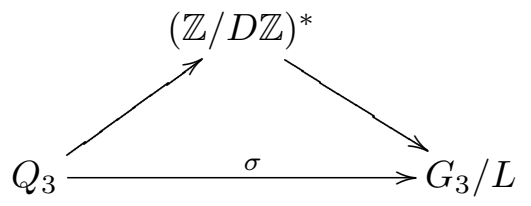

is well defined, and gives a commutative diagram of group homomorphisms. $G_{3} / L$ is arithmic.

With $G=G_{3}$, Theorem 1 is established.

Proof of the Corollary to Theorem 1. If integers $r, s$ divide $Q_{3}$ and satisfy $r \equiv s(\bmod D)$, then $r / s$ in $Q_{3} \mapsto 1$ in $(\mathbb{Z} / D \mathbb{Z})^{*} \mapsto$ identity in $G_{3} / L$. Under the canonical map $Q_{3} \rightarrow G_{3}, r / s$ is taken to an element in $L$. Therefore $(r / s)^{|L|}$ is taken to the identity of $L$, and so of $G ;(r / s)^{|L|}$ belongs to $\Gamma$. In other terms, $(r / s)^{|L|}$ has a product representation of the asserted type.

6. Concluding remarks. Any integer $m$ made up of primes not exceeding $N$, not dividing $N$ and not among the $q$, has a representation

$$
m^{|G|}=\prod_{p \in P}(N-p)^{e_{p}},
$$

with the $e_{p}$ integral. The order of $G$ may also be replaced by $\phi(D)|L|$, with $D$ from the arithmicity condition of $G / L$.

We can determine an effective upper bound for a set of representatives for $G / L$ in terms of $\delta$ and $\sum_{p \mid N} 1 / p$ only. We find $D$. Given $(s, D)=1$, a sufficiently strong version of Dirichlet's theorem on primes in arithmetic progression provides that

$$
\sum_{\substack{p \leq y,(p, N)=1 \\ p \equiv s(\bmod D)}} \frac{1}{p}>\frac{2 \log \log y}{3 \phi(D)}-\sum_{p \mid N} \frac{1}{p}>c_{1}(\delta),
$$

for $y \geq y_{s}=\max \left(c_{0}, \exp \exp \left(\frac{\phi(D)}{2} \sum_{p \mid N} \frac{1}{p}\right)\right)$, say. There is a prime $p<y_{s}$, not dividing $N$ and not a $q$, which maps onto the class $s(\bmod D)$. By varying $s$, the arithmicity of $G / L$ guarantees a complete set of representatives for $G / L$. Note that for a certain constant $c_{1}$ depending at most upon $\delta$, $y_{s} \leq \exp \left(c_{1}(\log \log N)^{2 \phi(D)}\right)$ uniformly in $s$.

When $P$ runs through all primes $p<N,(p, N)=1$, we expect there to be no exceptional primes $q$. There is a reasonable hope that the representatives for $G / L$ determined in the preceding manner all belong to $\Gamma$. In that case we could replace $|G|$ in (8) by $|L|$, which would then not exceed 4 . 
Let $Q(y)$ denote the number of exceptional primes $q$ not exceeding $y$. From Theorem 1, an integration by parts shows that

$$
\int_{2}^{N} \frac{Q(y)}{y^{2}} d y \leq c_{4}(\delta)<\infty,
$$

uniformly in $N$. In particular, if $0<\gamma<1$,

$$
\min _{N^{\gamma} \leq y \leq N} \frac{Q(y) \log y}{y} \int_{N^{\gamma}}^{N} \frac{d y}{y \log y} \leq c_{4} .
$$

The integral is $-\log \gamma$, and for a suitable value of $\gamma$, independent of $N$, $Q(y)<y(4 \log y)^{-1}$ for some $y$ in $\left[N^{\gamma}, N\right]$. Then $\left(\frac{1}{2} y, y\right]$ contains at least $y(8 \log y)^{-1}$ primes not dividing $N$, and not among the $q$. Let $m$ denote their product.

For all sufficiently large $N, m$ will lie in the interval $\left[\exp \left(N^{\gamma} / 16\right)\right.$, $\exp 2 N]$. Moreover, since each $N-p$ has at most $c_{5} \log N / \log \log N$ distinct prime factors, in any representation of the form (8),

$$
\sum_{p \in P}\left|e_{p}\right| \geq|G| y \log \log N\left(8 c_{5} \log y \log N\right)^{-1}>N^{\gamma}(\log N)^{-2}, \quad N \geq N_{2} .
$$

The generality of Theorem 1 militates against a reduction in the number of terms in the representing product.

Again let $P$ contain all the primes up to $N$ but not dividing $N$. To remove the exceptional primes $q$ in Theorem 1 in this case it would suffice to show that given a positive integer $d,(d, N)=1$, there is a prime $p$, not exceeding $N$, such that $p \equiv N(\bmod d),(N-p) d^{-1}$ is not divisible by any $q$. Since the $q$ might cover all primes in an interval $\left(N^{\varepsilon}, N\right]$, we are essentially to represent $N$ in the form $p+n$ where every prime divisor of $n$ is at most $N^{\varepsilon}$ in size. This is a problem of independent difficulty. Of course we need only solve it for a certain fixed $\varepsilon>0$, so there is some hope, involving much calculation.

The present paper provides the details to a lecture that I gave as the second plenary address on the first day of the international conference in analytic number theory held in Kyoto, May 19 to 25, 1996. The statement of Theorem 1 is a little complicated, and when $P$ is the set of all primes $p<N$, $(p, N)=1$, the presence of the exceptional primes $q$ does not seem intrinsic. At the end of that same day, my pleasure at being in Japan combined with jet lag to relax me, and I succeeded in devising a method to remove the exceptional primes. Of the various results possible, the following may be compared with Conjecture III.

TheOREM 2. There is an integer $k$ so that if $c>0, N>N_{0}(c)$, then every integer $m$ in the range $1 \leq m \leq(\log N)^{c},(m, N)=1$, has a represen- 
tation

with integral exponents $d_{p}$.

$$
m^{k}=\prod_{p \leq N / 2}(N-p)^{d_{p}}
$$

An explicit value can be given for $k$.

Although the proof of Theorem 2 proceeds from Theorem 1, considerable further argument is required, and I leave it to another occasion.

It is with great pleasure that I thank the organisers, Professors HirataKohno, Noriko, Motohashi, Yoichi and Murata, Leo, for the invitation to speak at this conference, for the financial help, and for their wonderful hospitality.

\section{References}

[1] P. D. T. A. Elliott, Probabilistic Number Theory I. Mean-Value Theorems, Grundlehren Math. Wiss. 239, Springer, New York, 1979.

[2] -, Arithmetic Functions and Integer Products, Grundlehren Math. Wiss. 272, Springer, New York, 1985

[3] -, Additive arithmetic functions on intervals, Math. Proc. Cambridge Philos. Soc. 103 (1988), 163-179.

[4] - The concentration function of additive functions on shifted primes, Acta Math. 173 (1994), 1-35.

[5] —, The multiplicative group of rationals generated by the shifted primes, I, J. Reine Angew. Math. 463 (1995), 169-216.

[6] W. J. Ellison et M. Mendès-France, Les nombres premiers, Hermann, Paris, 1975 .

[7] G. Halász, Über die Mittelwerte multiplikativer zahlentheoretischer Funktionen, Acta Math. Acad. Sci. Hungar. 19 (1968), 365-403.

[8] G. Pólya und G. Szegő, Aufgaben und Lehrsätze aus der Analysis I, Grundlehren Math. Wiss. 19, Springer, Berlin, 1925.

Department of Mathematics

University of Colorado at Boulder

Campus Box 395

Boulder, Colorado 80309-0395

U.S.A.

E-mail: pdtae@euclid.colorado.edu 